\title{
Making mundane pleasures visible: mediating daily likings with lightweight technology
}

\author{
Marije Kanis • Willem-Paul Brinkman
}

Received: 9 September 2008/Accepted: 2 July 2009/Published online: 16 January 2010

(C) The Author(s) 2010. This article is published with open access at Springerlink.com

\begin{abstract}
This article discusses the sharing of daily pleasures with lightweight technology. Two mobile applications called PosiPost Me (Mobile internet edition) and PosiPost Be (Bluetooth edition) were developed to understand the potential of remote and proximity-based sharing of positive messages. These implementations are compared through an empirical study of 15 participants in England and The Netherlands who used both applications for a week each. Drawing from interviews and a corpus of 379 shared messages, the study presents an exploration of the types of pleasures people expressed in everyday use. The study addresses the influence of the two applications on the nature of the messages shared. It further points to the importance of the mundaneness of the postings and discusses the role lightweight mobile technology can play in making mundane pleasures visible.
\end{abstract}

Keywords Mundane pleasures - Positive messaging · Mobile technology $\cdot$ Positive psychology ·

Lightweight interaction

This paper extends work previously presented at the SIMTech'08 workshop.

\footnotetext{
M. Kanis ( $₫)$

Brunel University, Kingston Lane, Uxbridge,

Middlesex UB8 3PH, UK

e-mail: amkanis@gmail.com

W.-P. Brinkman

Delft University of Technology, Mekelweg 4,

2628 CD Delft, The Netherlands

e-mail: w.p.brinkman@tudelft.nl
}

\section{Introduction}

Technology is shaping many aspects of people's lives, to such an extent that digital services and social applications have almost become inescapable. This technological proliferation can sometimes be experienced as oppressive, and thus can negatively contribute to people's wellbeing. One could posit that phenomena such as 'social network fatigue' $[1,2]$ are signs that there is a need for more lightweight interactions with technology; relatively simple applications (in terms of functionality) that do not demand a lot of commitment and personal effort from the user, in order to be sensitive to people's busy daily (social) life and practices.

Since the inception of the field of Human-Computer Interaction (HCI) much progress has been made on improving the usability of technology and (increasing) its functionality. Growing subfields such as affective computing [3], social computing and UX (user experience) design have shown that technology can emotionally connect and delight people, e.g. [ 4, 5]. If, according to Aristotle, the highest and most important goal for human beings is happiness [6], then the ultimate goal of technology could be seen as facilitating people's experience of positive affect. This does not simply imply the design of a fun technology or pleasant-to-use product, but a refocusing on understanding how technology can be designed for people to experience positive affect in their everyday life. There is clearly an opportunity to employ technology to uncover the nature of daily pleasures and to support the sharing of positive affect. In doing so, there might be a role for lightweight technologies to play. As mobile phones have become 'mundane' [7], meaning that they are increasingly pervasive and integrated in people's daily lives, they offer unique characteristics for sharing and recording people's 
day-in, day-out pleasurable moments, in a more lightweight, non-invasive way.

This work aims to address the challenge of lightweight interaction through the design and use of mobile applications that support humans in sharing the pleasures evoked by everyday life. Simple applications, running on mundane mobile technology, are presented as non-invasive interventions to record and share positive thoughts and pleasant experiences. In doing so, this work aims to contribute to the understanding of the mediation of positive affect that lightweight mobile technology could support.

\subsection{Related work}

Designing lightweight technologies and applications that have positive emotional benefits is a relatively new challenge for the HCI community. A better understanding of people's positive emotions and pleasures in everyday life that goes beyond the mere encounter with technology is needed. The field of positive psychology [8], which focuses on mental wellness, rather than illness, directly concerns understanding positive affect and human wellbeing in everyday life. A small number of researchers in HCI and its related fields have already drawn inspiration from positive psychology for a more explicit positive approach to human-centred design and evaluation [9], for UX research with a focus on designing (engaging) systems for fun or pleasure [10], or for preliminary design theories that are based on supporting human needs and flourishing [11]. So far however, most inspiration and research efforts have come from the 'flow theory', one of the key concepts of positive psychology that was originally coined by Csikszentmihalyi [12] and is predominantly used for the goal towards creating engaging or optimal experiences with technology, e.g. [13]. However, the flow state does not cover all moments when people experience pleasure in everyday life. For example, when someone rewards herself with a nice cup of tea after a laborious task. Moreover, supporting people's experience of positive affect or wellbeing does not simply imply the design of an engaging technology or pleasant-to-use, aesthetic product. This research is taking a different approach in its (psychotherapeutic) aim to use technology for improving the experience of positive affect in everyday life. In doing so, this work builds on empirical studies from positive psychology that have shown the benefits of experiencing positive affect [14] and positive disclosure. King [15] for example, found health benefits in writing about positive emotional goals, while others [16] found that practicing grateful or positive thinking through simple exercises-in particular activities in which participants are asked to recall and write down good things that happened that day [17]—can enhance wellbeing. Thus, a body of research in positive psychology suggests that exercises concerning expressing and cultivating positive thoughts can be a simple yet powerful way of improving a person's wellbeing. However, the role technology could play in such positive psychology interventions remains largely unexplored. To address this challenge, this paper presents two lightweight mobile applications, designed for the activity of expressing and sharing daily pleasures, to understand the potential of remote and proximity-based sharing of positive messages.

\section{Design rationale}

Building on positive psychology research, two mobile messaging technologies PosiPost Me (a Mobile internet edition) and PosiPost Be (a Bluetooth edition) were developed to investigate the potential of mediating positive affect through technology with people over a distance (Me) and in close proximity $(\mathrm{Be})$. The reason for two applications was to study and compare the influential role of these different media through analysis of (a) contextual cues in use and (b) the nature of the messages shared.

\subsection{Lightweight mobile technology}

The two mobile prototypes were built to run on mundane technology to facilitate a straightforward way for expressing and sharing daily likings. The design of the technology was based on previous studies that had pointed towards a mobile [18] and simple, lightweight technology [19] in expressing positive thoughts. The motivation for addressing lightweight interaction was also a direct response to technological developments that have led to provide substantially more functionality to the user, sometimes leading to heavily featured, 'bloated' software that is increasingly complex to use [20]. Furthermore, some (social) software are sometimes seen as a burden due to the social expectations to reciprocate [21].

The design of PosiPost addresses the lightweight challenge in three ways:

1. The applications are built on top of mundane, mobile technology, for the reason that mobile devices could be considered generally well adopted and integrated in people's current practices;

2. The designed applications afford anonymous disclosure. PosiPost enables the user to write and share messages about positive moments anonymously to promote higher levels of self-disclosure, and because of a lack of identifiability and accountability, anonymous users will be less likely to experience pressure or demanding expectations from others [22]; 
3. PosiPost affords a simple micro-blogging functionality. The increasing popularity of tools with micro-blogging features, a form of blogging that allows users to send brief text status, such as Twitter and Facebook updates, indicate an increasing interest in more simple forms of self-expression.

When creating a message with PosiPost, the user is asked to briefly describe a positive moment by finishing the sentence starting with the prefix: "Today, I like". Previous studies had already shown the potential for such a prefixbased elicitation of positive emotions [23].

\subsubsection{PosiPost Me}

PosiPost Me (Fig. 1) is a simple client-server based application that enables the sharing of messages starting with the prefix 'Today, I like', via mobile Internet.

The distribution of the messages is completely randomized and anonymous, so that the context and origin (location, time and messenger) of the postings are not known to the receiver. Retrieval is based on message pull: anytime the user likes, (s)he can ask the client to retrieve a posting.

\subsubsection{PosiPost Be}

With the other mobile version, PosiPost Be (shown in Fig. 2), users are able to automatically exchange messages with other posiposters who come in close proximity, via Bluetooth. In this design, users have the option to reveal clues about their identity and origin (such as an alias or a postcode). Due to the proximity, it is easier to know the context (time, place and the possible sender) of the posting. With mobile phones equipped with PosiPost Be software, users are able to share positive thoughts from nearby users directly without connection to a network or server. The sharing of the messages is thus based on a decentralized peer-to-peer system, which uses a push method for receiving the messages. Users can moderate the postings,

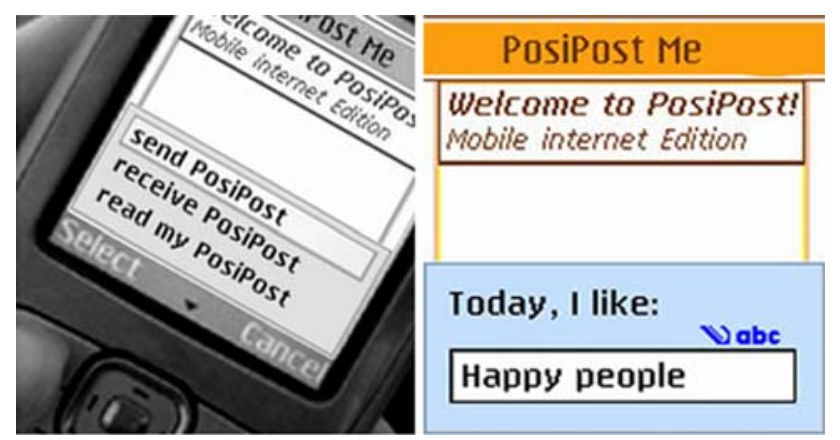

Fig. 1 Sending a message with PosiPost Me (Mobile internet edition)

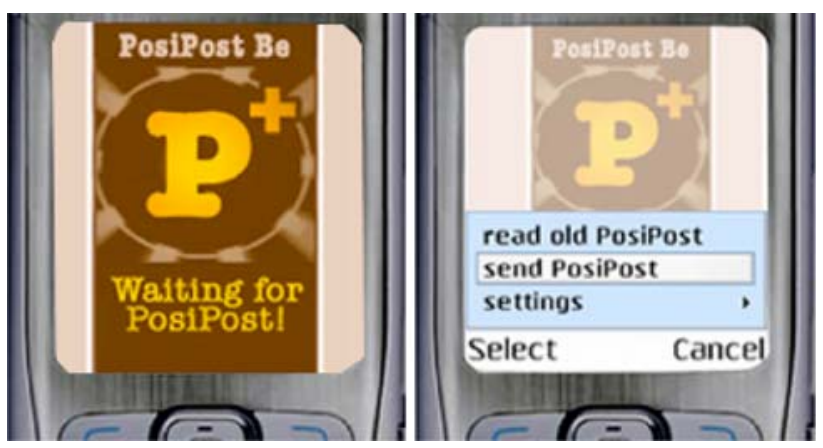

Fig. 2 Waiting for and sending a message with PosiPost Be (Bluetooth edition)

by having the option to pass on the received messages they like. In terms of the system development and Bluetooth's automated sending functionality in PosiPost $\mathrm{Be}$, the application was far more complicated to build than PosiPost's Mobile internet edition. However, both versions offer the same functionality to receive and send positive messages, while having different contextual levels. By having two different applications, it is possible to compare (i) whether contextual cues and anonymity are important for mediating positive affect and (ii) whether remote or proximity-based posting influences the contents of the messages shared.

\section{Study}

The technology was developed as part of an empirical study, which had three main purposes: (1) to study the kind of (mundane) daily pleasures shared with the technology and test its potential positive and mundane nature; (2) to understand the potential influence of the medium (and different levels of proximity and anonymity) on usage and the content of the messages shared; (3) to find out what happens when people are given the opportunity to anonymously record and share feelings with others using lightweight technology.

\subsection{Method}

The study involved 15 participants, 8 male and 7 female, who were 20-40-year-old ( $M=28, \mathrm{SD}=3$ ) post-graduates. The participants had a wide variety of different nationalities, but were based in England and The Netherlands. The users in England belonged to a group of friends living near each other in London. In The Netherlands, the users were colleagues who worked in the same university building in Delft. These sites were chosen to ensure that messages would be regularly exchanged with people in close proximity, when using PosiPost's Bluetooth edition. When the participants 
were asked about their mobile phone experience, $20 \%$ said they used mobile Internet, $80 \%$ used their phone just to call and for SMS, and all of them indicated to have a mobile phone.

Both PosiPost applications were given to participants and they were informed that they could use them when, where and how they wanted to for 1 week each. This was to promote usage in the context of normal daily practices and routines so to bring about genuine and real posting behaviour, without the participants feeling observed. Each participant group received the applications in a different order to counterbalance any possible learning and habituation effects. Furthermore, a neutral stop week was held to reduce this effect even further. The postings shared with both versions were recorded for later analysis and comparison. Furthermore, all participants were interviewed after using both versions of PosiPost and considered both created and read messages.

\subsection{Content analysis}

A content analysis [24, 25] was conducted on the postings that were created with the two different versions of PosiPost (e.g. "Today, I like ice skating! On natural ice"). This meant that the posted messages were analysed and coded into different categories to address the question of what the participants' shared expressions of happy moments were about and to explore the potential mundaneness of those. All the categories are displayed in Table 1, along with examples from the collected posipostings (see [23] for further details on the categories). Two coders (the first author and an autonomous coder) did the coding of the postings independently in order to ensure reliability. Afterwards, the consistency of the allocation of the codings by the two coders was examined by calculating a series of Cohen's Kappa, a measure for inter-rater reliability. For all categories, there was a high level of agreement between the coders, given that all Cohen's Kappa values were above 0.75 , which is normally considered as excellent [25]. In the few cases where the two coders initially disagreed, they discussed and agreed on a unified classification afterwards. The categories were based on the findings from a previous study [23] and Tiger's pleasure framework [26]. This framework describes four distinct pleasures (psycho-pleasure, ideo-pleasure, socio-pleasure and physio-pleasure) and illustrates a broader range of classifications for pleasure when compared to other pleasure frameworks [12, 27]. Its utility to classify and understand distinct types of pleasure has already been demonstrated [4].

The categories were designed not to be mutually exclusive. For example, a posting such as "Today, I like having cake with my colleagues" could be classified as a socio-pleasure and further be placed in other categories such as mundane and physio-pleasure. Thus, each posting could have multiple codings. All the 379 postings could be coded in at least one of all the categories each, suggesting that this set of categories (all displayed in Table 1) is describing all postings in some way. As the vast majority of the postings $(99 \%)$ fell in at least one of the mundane or pleasure categories (i.e. mundane, or pleasure category), the focus of the analysis was therefore on these categories.

\section{Results}

The results of the investigation address the three aims that were earlier outlined in the description of the study. This means that the content analysis offers insights into the various pleasures people take from life, while focusing on their positive and mundane nature. Furthermore, the results of the study provide insights into the contextual use of the technology and its role in encouraging positive affect.

\subsection{Mundane pleasures}

The study resulted in $379(179+200)$ messages created with PosiPost's two editions (Mobile internet and Bluetooth, respectively). The results of the content analysis are displayed in Table 1, along with examples from the collected postings. These address the first aim of the study: to provide insights in the kind of (mundane) daily pleasures shared with the technology and test its potential positive and mundane nature.

The content analysis was to address the first aim of the study, which was to study the kind of (mundane) daily pleasures shared and to test its potential positive and mundane nature. Three immediate conclusions can be drawn from this content analysis. Firstly, all postings were considered as positive and non-offensive. Naturally, users' positive usage needs to be seen in the critical context of a study setting as this may have influenced participants' posting behaviour. However, this study affirms the findings of a previous studies (including one in which participants did not know that the contents they created would be analysed for the purpose of a study) that positive emotions can be mediated by (mobile) technology [23]. Secondly, from the four pleasure categories, Psycho-pleasure scored lowest (21\%). Even postings referring to Leisure time with its $24 \%$ scored slightly higher. This can be seen as an indication that the pleasures derived from active (flow) states should not be the only 'pleasure concept' for the HCI community to focus on. Thirdly, a very high proportion of codings fell in the Mundane category. And thus, the study suggests that messages of a positive and mundane nature can be shared. However, it needs to be noted that the setting and participants' awareness of a study could have influenced the content of the messages. Consequently, this paper calls for 
Table 1 Numbers and percentages of postings according to content category

\begin{tabular}{|c|c|c|c|c|}
\hline \multirow[t]{3}{*}{ Content category } & \multicolumn{4}{|c|}{ Posted content that falls in category } \\
\hline & \multirow{2}{*}{$\begin{array}{l}\mathrm{Me} \\
\%\end{array}$} & \multirow{2}{*}{$\begin{array}{l}\mathrm{Be} \\
\%\end{array}$} & \multicolumn{2}{|c|}{ Total PosiPost Me and $\mathrm{Be}$} \\
\hline & & & Freq & $\%$ \\
\hline $\begin{array}{l}\text { PSYCHO-PLEASURE (progress, accomplishment): enjoyment derived from a certain } \\
\text { progress, accomplishment or flow. Can refer to the satisfaction enjoyed as a result } \\
\text { of individually motivated tasks or acts. } \\
\text { "Today, I completing a jigsaw puzzle" }\end{array}$ & 25 & 17 & 78 & 21 \\
\hline $\begin{array}{l}\text { IDEO-PLEASURE (personal values, attitudes): pleasure derived from books, art } \\
\text { and music etc. that are in agreement with attitudes and personal aesthetic, political, } \\
\text { ecological values. } \\
\text { "Today, I like what I do for a living" }\end{array}$ & 30 & 23 & 101 & 27 \\
\hline $\begin{array}{l}\text { SOCIO-PLEASURE (human, social): pleasure derived from social contact } \\
\text { and relationships with others. } \\
\text { "Today, I like getting to know my colleagues better" }\end{array}$ & 41 & 33 & 140 & 37 \\
\hline $\begin{array}{l}\text { PHYSIO-PLEASURE (physical sensation): pleasure derived from haptic, visual, audio } \\
\text { senses. Can e.g. refer to the physical sensation obtained from eating or drinking. } \\
\text { "Today I like the taste of chocolate melting in my mouth" }\end{array}$ & 47 & 46 & 176 & 47 \\
\hline $\begin{array}{l}\text { MUNDANE (everyday event): pleasure derived from a mundane, ordinary, simple } \\
\text { thing happening in life. } \\
\text { "Today, I like working at home" }\end{array}$ & 60 & 65 & 238 & 63 \\
\hline Negative: posting is not about something positive & 0 & 0 & 0 & 0 \\
\hline Offensive: posting could be regarded as racially, sexually or politically offensive & 0 & 0 & 0 & 0 \\
\hline $\begin{array}{l}\text { Leisure time (break, rest): posting contains task-free or relaxation moment. } \\
\text { "Today, I like the weekend!" }\end{array}$ & 33 & 16 & 91 & 24 \\
\hline $\begin{array}{l}\text { Situatedness (right here, right now): a posting concerning what is happening } \\
\text { at particular moment in time and place } \\
\text { "Today, I like writing the best posipost" }\end{array}$ & 18 & 21 & 289 & 76 \\
\hline $\begin{array}{l}\text { Emotion (explicit): an explicit reference to an emotional state of a person } \\
\text { "Today, I like happy people" }\end{array}$ & 3 & 6 & 17 & 4 \\
\hline $\begin{array}{l}\text { Overcoming negative emotions or experiences } \\
\text { "Today, I like going to return the washing machine" }\end{array}$ & 6 & 4 & 18 & 5 \\
\hline $\begin{array}{l}\text { Materialistic possession (novelty) } \\
\text { "Today, I like my new outfit" }\end{array}$ & 16 & 6 & 37 & 10 \\
\hline $\begin{array}{l}\text { Activity: a reference to an action } \\
\text { "Today, I like playing football" }\end{array}$ & 24 & 13 & 68 & 18 \\
\hline $\begin{array}{l}\text { Time: a reference to time } \\
\text { "Today, I like that it's almost xmas" }\end{array}$ & 22 & 16 & 72 & 19 \\
\hline $\begin{array}{l}\text { Object (medium): a reference to a physical object } \\
\text { "Today, I like the phone" }\end{array}$ & 24 & 17 & 78 & 21 \\
\hline $\begin{array}{l}\text { Environment (location): a reference to a physical location or posting contains } \\
\text { an environmental factor, such as a reference to the weather. } \\
\text { "Today, I like the cold weather" }\end{array}$ & 30 & 23 & 100 & 26 \\
\hline
\end{tabular}

further investigation and discussion that could potentially guide design and research efforts for everyday life in HCI.

\subsection{PosiPost Be versus PosiPost Me}

The created contents of both the applications were analysed and compared to address the second aim of the study, i.e. to understand the potential influence of the application used. In comparing the message contents of the presented applications (PosiPost Be or PosiPost Me) the differences were mostly subtle. The analysis of the study investigating the role of the presented media, showed that PosiPost Be and PosiPost Me can both function as a medium for positive expression; independent of whether posting is proximity-based or over longdistance, they can both provide a vehicle for projecting 
into someone else's daily lives and engage with another person's positive glances.

Pearson's chi-square tests were carried out to compare the codings of the messages generated with PosiPost $\mathrm{Me}$ versus PosiPost $\mathrm{Be}$ for the pleasure and mundane categories in order to understand the potential influence of the version used. No significant differences $(p>0.05)$ were found for these categories, indicating that the mobile version used had no clear influence on the kinds of pleasures and mundaneness reported. However, significant differences were found for the categories activity $\left(\chi^{2}=7.14\right.$, $d f=1, p=0.008)$, leisure time $\left(\chi^{2}=15.10, d f=1\right.$, $p<0.001)$ and materialistic possession $\left(\chi^{2}=8.83\right.$, $d f=1, p=0.003)$. Thus, the version used might have had some influence on the posted content related to these categories. For all of these three categories, significantly more of this kind of posted content was generated with PosiPost Me than with PosiPost Be.

The length of the messages, in amount of words per posting, for PosiPost $\mathrm{Me}(M=3.5)$ and PosiPost Be $(M=3.1)$ was not significantly dependent on the used version of PosiPost $(t(378)=-1.53, p=0.13)$. The only other found statistical significant difference between PosiPost $\mathrm{Me}$ and $\mathrm{Be}(t(378)=-5.60, p<0.001)$ was that messages with PosiPost Me had more content codings associated with each posting. Thus, posted content created with PosiPost Me on average fell into more different categories than when created with PosiPost Be. This could be linked to the result that the categories activity, leisure time, and materialistic object had significantly more postings created with PosiPost Me. As the context of the messages with PosiPost Me is completely unknown, a speculative explanation for this could be that users were more diverse and descriptive in their postings to explain their current context. For example, postings such as "Today I like Acda $\&$ de Munnik" (a well-known band in Holland) or "Today I like colaquium" (a regular informal social meet-up in which the colleagues were having a Cola in Delft University's canteen) were less descriptive and explanatory than a posting such as "Today I like the cup of strawberry tea from my colleague". In the former cases it was possibly felt that a context did not really need to be established with the proximity-based sharing of postings with PosiPost Be. Some participants noted that they liked messages from countries that were different from their own, as they said they already knew about their own context (e.g. "Today I like trains and scenery in India"). Yet, they also said that they particularly liked the ones that they could somehow relate to their own context and so become positively inspired by. For example, when a participant initially felt annoyed because of being cold, she was cheered up by receiving the postings "Today I like have a hot chocolate" and "Today I like some glühwein?! To warm a bit" as she felt that these were good suggestions. Generally, in the interviews participants also said to particularly like funny postings such as "Today I like running around like a madman (better than being bored)".

On PosiPost Be, a participant noted that when other posiposters were around, more pressure was felt to make an interesting posting. On the other hand, PosiPost $\mathrm{Be}$, as a social proximity application, opens up interesting possibilities for social interaction in real space. For example, one participant noted: "I think PosiPost can add something to the usual conversations I have with colleagues here, as these are normally quite superficial. When I receive an interesting message I can strike up a conversation about this with a colleague." Observations about such a thing happening included one male poster discovering another female posiposter and asking his colleague: "Ah, were you the one liking pink dresses?" In an encounter between spatially proximate people, as facilitated by PosiPost Be, social interactions can be more focused and contextualized, as opposed to PosiPost Me. An example of this happening was one participant posting: "Today, I like looking forward to xmas dinner tomorrow" and then another co-located person send the posting "Today I like knowing you'll come to xmas dinner too." Also a posting generated with PosiPost Be, such as "Today, I like getting to know my colleagues better" could be seen as support for PosiPost Be by having more potential as a social icebreaker. However, participants seemed to be more interested in the posipost than the posiposter, and seemed to like to keep some social distance. As a participant noted: "The main important thing for me is still the posipost message." Also, PosiPost Be's function to reveal more about a person's identity (e.g. their origin) was rarely used. Participants stated that they preferred to remain anonymous and liked the ambiguous nature of decontextualized postings.

When using the lightweight PosiPost applications, the effort required and social expectations placed on users were relatively low as users were willingly, but 'minimally connected [19]' to other posiposters via a simple text stream. This practice unmasked an interesting tension, which concerns the desired levels of social interaction versus the required personal input when using technology. Studies, e.g. [28] show that when people experience positive affect, this can trigger an upward spiral that augments social bonding in everyday life. Therefore, the PosiPost applications offer a lot of potential to catalyse social interactions with other people, particularly in physical space. And so, the realization of cross-media communications with versions of PosiPost, such as moving on from on-line interactions towards face-to-face interactions, deserves further attention. 


\subsection{Encouraging positive affect}

The results of the study address the final aim of uncovering what happens when people are given the opportunity to share feelings by means of lightweight technology, particularly in terms of encouraging positive affect. The results of the content analysis suggest that positive thoughts of a predominantly mundane nature can be shared and mediated by mobile technology. The data from the interviews are also promising, as participants' accounts indicate that lightweight technologies, such as PosiPost can aid people in becoming more positive. This was illustrated by a Dutch participant who noted: "Even though when I wasn't in a positive mood I liked sending posipostings. Especially then, I enjoyed thinking about what I liked today in order to put myself in a better mood." Another participant in England noted: "I didn't send messages only when I was happy. On the contrary, like last week I felt sad and depressed because I didn't have a normal Internet connection, so I definitely also used it when I wasn't so happy to cheer me up." PosiPost, as a lightweight communication technology for shared positive disclosure, not only helped to uncover the benefits of expressing emotions, as is typically studied [15], but also emphasized the interesting potential and benefits of receiving the positive thoughts of others. Receiving messages with PosiPost Me and PosiPost Be happened much more frequently than creating postings, and messages were thus read more than written. The reasons users gave for this in the interviews was to really like receiving postings also due to the lower efforts required, which in the case of PosiPost Be even happened automatically. On the receiving end, retrieving a 'mundane' positive posting often seemed to inspire the receiver to reflect on his or her feelings in a more interesting, positive context.

\section{Implications for design}

The results of the study contribute to understanding the role lightweight technology can play in mediating mundane pleasures and making positive moments visible. The key implications concerning the design of mundane, lightweight technologies are outlined and discussed below.

\subsection{Making the invisible visible}

Weiser [29] and his vision of 'invisible computing' began with an interest in the role of computers in everyday life, and a desire to build computers that did not interfere with people's everyday activities. According to Weiser, a good tool is an invisible tool. By invisible, he meant that the tool does not intrude on people's consciousness, in which the focus is on the task, not the tool. Mundane technology links to this view by being so tightly entwined with activity and interaction in everyday practices, as to be almost invisible. In this research, the reason for using mundane mobile technology was to provide users with an invisible lightweight vehicle for the contrasting purpose of making everyday pleasures and their mundane nature explicitly visible. Thus, mundane technology was used to seamlessly support the person's capability to notice (i.e. make visible) pleasant situations in everyday life through the (invisible) PosiPost technology.

However, it should be noted though that $5 \%$ of the postings were related to the medium used or the PosiPost process, such as "Today I like using posipost with my n95 phone". Thus, although these were positive accounts, it indicates that users were sometimes consciously aware of the medium. This could therefore be interpreted as the PosiPost technology not entirely being invisible, or embedded in the background. Interestingly, the participants pointed out an issue with what can happen if a technology becomes 'too invisible'. In the interviews, some participants said that although wanting to PosiPost, they sometimes just forgot about it, as especially PosiPost Me as a pull medium was very much designed to be in the background. However, some participants said that seeing the actual phone reminded them of wanting to post. As further expressed by one participant: "Every time I saw the phone, I remembered, and then created and shared a couple of posipostings." Thus, a mobile phone, through its form factor may remind people about PosiPost, but it is the application running on it that makes people's positive thoughts visible. What could be learned is that even though making technology or an application invisible is a good objective, the potential consequence is that an application can become so lightweight, so mundane and so integrated in people's lives that it can completely disappear in the background, and thus difficult to notice or to be pleasantly surprised by. Thus designers should be aware of creating technology that becomes too invisible, as so that people do not forget that it can be used to support them.

\subsection{Mundane awareness}

This paper contributes to the discussion of mundane technology and lightweight design by demonstrating how lightweight applications built on top of mundane technology, could contribute to users' positive affect in everyday life. The design of PosiPost seems to increase users' awareness of positive moments in daily life-something that is, due to its mundaneness, often originally invisible. Through a simple disclosure of this awareness, other users can in turn adapt the same remarks to their interpretations of everyday life. By describing the design of PosiPost, it 
demonstrated how the lightweight design challenge can be addressed for making positive moments visible. The approach taken to the lightweight design of PosiPostsimply supporting people to construct and become aware of mundane pleasures-helps to go beyond the 'wow' experience rhetoric that treats user experience as a mere encounter with the technology, detached from the context, happenings and demands in everyday life. This approach implies that technology developers are encouraged to step back, and create lightweight technology platforms that are simple enough for empowering users to create something positive out of the mundane themselves.

\section{Conclusion}

In this article, mundaneness has been addressed in two ways: (1) to describe technology, and (2) to classify the generated content. The presented lightweight applications, built on top of mundane mobile technology seem to support the person's capability of becoming aware of pleasant situations in everyday life. The study and content analysis provided insights into the pleasures people make out from ordinary happenings in life, and uncovered their particular mundane character. The investigation of the message contents of PosiPost Be versus PosiPost $\mathrm{Me}$ showed that, although different in contextual considerations, both can function as a medium for positive expression, independent of whether posting is proximitybased or over long distance. The results support the hypothesis that users are more diverse and descriptive in explaining their current context when they create longdistance postings compared to proximity-based postings. Furthermore, the study showed that both the presented PosiPost versions and their ability to support shared anonymous disclosure offers interesting potential in terms of aiding people to become aware of positive moments. Although needing further inquiry, the applications may even prove useful as a therapeutic exercise. By designing PosiPost as a lightweight technology for shared positive expression, the presented study uncovered various pleasures in everyday life that people engage in. In doing so, it demonstrated an opportunity for the development of lightweight technological solutions for supporting and encouraging positive disclosure. It could be concluded that the strength of the presented lightweight technology did not lie in the abundance of complex 'wow' functionalities, but in the rich mundane contents and notifications shared and created by its users.

Acknowledgments We thank Antti Salovaara, Mark Perry, Iftikhar Kahn, Niall Winters and the study participants.
Open Access This article is distributed under the terms of the Creative Commons Attribution Noncommercial License which permits any noncommercial use, distribution, and reproduction in any medium, provided the original author(s) and source are credited.

\section{References}

1. McFredies P (2007) Technically speaking: Tired vs. Wired. Spectrum, IEEE 44:68

2. Goth G (2008) Are social networking sites growing up? Distributed systems online. IEEE 9:1-3

3. Picard R (1997) Affective computing. MIT Press, Cambridge

4. Jordan P (2000) Designing pleasurable products. Taylor and Francis, London

5. Sundström P, Ståhl A, Höök K (2005) eMoto-a user-centred approach to affective interaction. In: Proceedings of ACII'05, Lecture Notes in Computer Science. Beijing, China

6. Aristotle (350 BC) Nicomachean ethics. Internet Classics Archive

7. Graham C, Rouncefield M (2007) In: Proceedings of the workshop on social interaction and mundane technologies, Melbourne, Australia

8. Seligman M, Csikszentmihalyi M (2000) Positive psychology: an introduction. Am Psychol 55:5-14

9. Sklar A, Gilmore D (2004) Are you positive? Interactions 11:2833

10. Hassenzahl M, Tractinsky N (2006) User experience-a research agenda (Editorial). Behav Inform Technol 25:91-97

11. Zhang P (2007) Toward a positive design theory: principles for designing motivating information and communication technology. In: Avital M, Bolland R, Cooperrider D (ed) Designing information and organizations with a positive lens. Elsevier, Amsterdam, pp 45-74

12. Csikszentmihalyi M (1990) Flow: the psychology of optimal experience. Harper and Row, NY

13. Bederson B (2004) Interfaces for staying in the flow. Ubiquity 5:1

14. Lyubomirsky S, King L, Diener E (2005) The benefits of frequent positive affect: does happiness lead to success? Psychol Bull 131:803-855

15. King L (2001) The health benefits of writing about life goals. Personal Soc Psychol Bull 27:798-807

16. Emmons R, McCullough M (2003) Counting blessings versus burdens: an experimental investigation of gratitude and subject wellbeing in daily life. J Pers Soc Psychol 84:377-389

17. Seligman M, Steen T, Park N, Peterson C (2005) Positive psychology progress: empirical validation of interventions. Am Psychol 60:410-421

18. Kanis M, Brinkman W-P, Perry M (2009) Designing for positive disclosure: what do you like today? Int J Industrial Ergonom 39:564-572

19. Kanis M, Perry M, Brinkman W-P (2008) Minimal connectedness: exploring the effects of positive messaging using mobile technology. CHI ' 08 extended abstracts on human factors in computing systems, pp 2513-2522

20. McGrenere J, Moore G (2000) Are we all in the same "bloat"? Proceedings of graphics interface 2000, pp 187-196

21. Salovaara A (2008) Struggling with gift-giving obligations: when mobile messages are too laborious to reciprocate. In: Proceedings of HCI 2008, pp 83-86

22. Joinson A (2001) Self-disclosure in computer-mediated communication: the role of self-awareness and visual anonymity. Eur J Soc Psychol 31:177-192 
23. Kanis M, Brinkman W-P (2007) What do people like? The design of a mobile tool to harness and share positive thoughts. In: Proceedings of the 14th European conference on cognitive ergonomics, pp 191-198

24. Coolican H (1994) Research methods and statistics in psychology. Hodder and Stoughton, London

25. Robson C (1993) Real world research: a resource for social scientists and practitioner-researchers. Blackwell, Oxford
26. Tiger L (1992) The pursuit of pleasure. Transaction, London

27. Wen-chih C, Tyan Yu W (2007) Exploring types and characteristics of product forms. Int J Design 1:3-14

28. Waugh C, Fredrickson B (2006) Nice to know you: positive emotions, self-other overlap, and complex understanding in the formation of new relationships. J Positive Psychol 1:93-106

29. Weiser M (1994) The world is not a desktop. Interactions 1:7-8 